\title{
First harmonic injection locking of 24-GHz-oscillators
}

\author{
M. R. Kühn and E. M. Biebl \\ Technische Universität München, Fachgebiet Höchstfrequenztechnik, Arcisstr. 21, 80333 München, Germany
}

\begin{abstract}
An increasing number of applications is proposed for the $24 \mathrm{GHz}$ ISM-band, like automotive radar systems and short-range communication links. These applications demand for oscillators providing moderate output power of a few $\mathrm{mW}$ and moderate frequency stability of about $0.5 \%$.

The maximum oscillation frequency of low-cost off-theshelf transistors is too low for stable operation of a fundamental $24 \mathrm{GHz}$ oscillator. Thus, we designed a $24 \mathrm{GHz}$ first harmonic oscillator, where the power generated at the fundamental frequency $(12 \mathrm{GHz})$ is reflected resulting in effective generation of output power at the first harmonic. We measured a radiated power from an integrated planar antenna of more than $1 \mathrm{~mW}$. Though this oscillator provides superior frequency stability compared to fundamental oscillators, for some applications additional stabilization is required.

As a low-cost measure, injection locking can be used to phase lock oscillators that provide sufficient stability in free running mode. Due to our harmonic oscillator concept injection locking has to be achieved at the first harmonic, since only the antenna is accessible for signal injection. We designed, fabricated and characterized a harmonic oscillator using the antenna as a port for injection locking. The locking range was measured versus various parameters. In addition, phase-noise improvement was investigated. A theoretical approach for the mechanism of first harmonic injection locking is presented.
\end{abstract}

\section{Introduction}

Injection locking is a low-cost measure to phase lock oscillators that provide sufficient stability in free running mode. In our case, injection locking has to be achieved at the first harmonic, since only the antenna is accessible for signal injection. Thus, the coupling circuit is integrated in the planar antenna structure. We investigated the locking range, i.e. the acceptable deviation between free-running oscilla-

Correspondence to: M. R. Kühn (kuehn@ei.tum.de) tion frequency and frequency of the synchronization source versus different parameters like output power of the oscillator and power of the synchronization source. Moreover, phasenoise improvement due to injection locking was investigated. The mechanism of first harmonic injection locking and the resulting dependencies will be discussed.

\section{Oscillator design}

The oscillator design is based on the one port approach. The fundamental wave is at $12 \mathrm{GHz}$ and reflected at the filter, i.e. there is no power delivered at the fundamental frequency. The oscillating conditions are fulfilled at $12 \mathrm{GHz}$, but the output signal is the first harmonic at $24 \mathrm{GHz}$. Because we do not take out any power at $12 \mathrm{GHz}$ the first harmonic is generated efficiently. Spurious spectral components, namely the fundamental and the higher harmonics are suppressed by use of a filter (Fig. 1). We measured an output power of about $3 \mathrm{dBm}$ at $50 \Omega$ behind the filter. The oscillator is designed for maximum power at $24 \mathrm{GHz}$ and ideal oscillating conditions at $12 \mathrm{GHz}$.

The bias network in this design has to block both $12 \mathrm{GHz}$ and $24 \mathrm{GHz}$. Therefore, two radial stubs were attached at each DC-line. The small stub is designed for $24 \mathrm{GHz}$ and the big stub is for $12 \mathrm{GHz}$ and for $24 \mathrm{GHz}$. The Design is realised in microstrip-technology on RT-Duroid.

To radiate the $24 \mathrm{GHz}$ wave the oscillator is coupled to a common rectangular patch antenna, see Fig. 1. The patch is a $\lambda / 2$ resonator. At the other side of the antenna a coupling structure is connected. Here the $24 \mathrm{GHz}$ reference signal is injected into the oscillator via the antenna and the filter. The coupling line is realised as $100 \Omega$ line to reduce the influence to the antenna. Furthermore a coupling slot is inserted, which allows for relatively weak coupling between the reference source and the resonant patch antenna in order to provide a sufficiently high loaded q-factor of the antenna. The simulated insertion loss of this coupler is $8.45 \mathrm{~dB}$ at $24 \mathrm{GHz}$. 


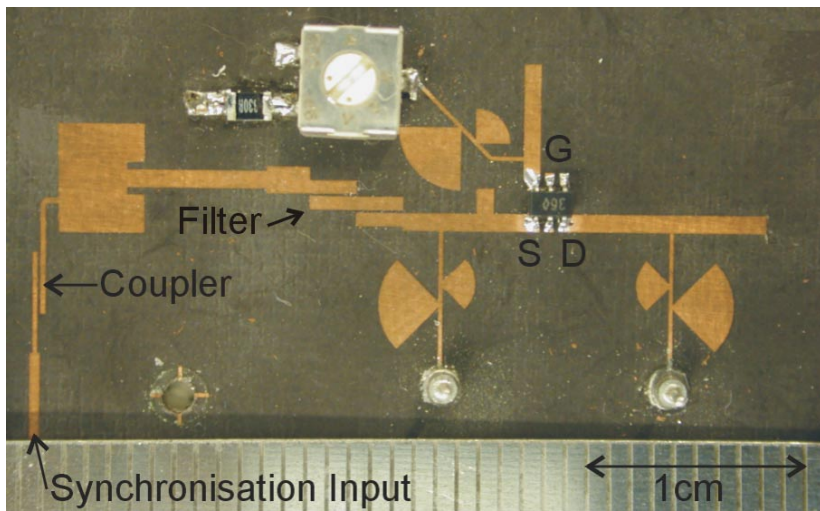

Fig. 1. Free running harmonic Oscillator with patch antenna, filter and coupler.

\section{Injection locking}

Injection locking is the synchronisation in frequency and phase of a free running oscillator with a source. The mechanism of injection locking has been observed in a wide variety of oscillators (Adler, 1946; Kurokawa, 1973; York, 1993; Navarro and Chang, 1996). It can be shown, that the output phase of a simple oscillator with an injected signal is given by Adler's equation (Adler, 1946).

$$
\frac{d \varphi}{d t}=\omega_{0}-\omega_{i n j}+\underbrace{\frac{\rho}{\alpha} \frac{\omega_{0}}{2 Q}}_{\Delta \omega_{m}} \sin \underbrace{(\psi-\varphi)}_{\Delta \varphi}
$$

where $\omega_{0}$ is the free-running frequency, $\omega_{i n j}$ is the injected signal, a is the free-running oscillating amplitude, $Q$ is the quality-factor of the oscillator's resonant circuit, $\rho$ is the amplitude of the injected signal measured at the free-running oscillator. For phase synchronising the oscillator with the reference signal a steady state solution $d \varphi / d t=0$ has to exist. Solving (3.1) for steady state gives

$\Delta \varphi=\sin ^{-1}\left(\frac{\omega_{i n j}-\omega_{0}}{\Delta \omega_{m}}\right)$

which shows that an injection locked solution is possible only when the injected signal frequency lies within the "locking range" of the oscillator $\omega_{0} \pm \Delta \omega_{m}$. Solving (3.2) will result in two possible solutions because of the inverse sine. Here a stability analysis is necessary to gain the right solution. The phase is distort from its free-running state $\left(\varphi_{0}\right)$ by writing $\varphi=\varphi_{0}+\delta \varphi$, which reduces (3.1) to

$\frac{d \delta \varphi}{d t}=-\delta \varphi \Delta \omega_{m} \cos \Delta \varphi$

The distortion will decay in time provided that $\cos \Delta \varphi>0$, which restricts the phase difference to the range $-\pi / 2 \leq$ $\Delta \varphi \leq \pi / 2$. The locking range is proportional to the injected signal amplitude and inverse proportional to the Q-factor and the oscillator's amplitude. To get a large locking range in a practical system, low-Q oscillators, with large injected signals are required.

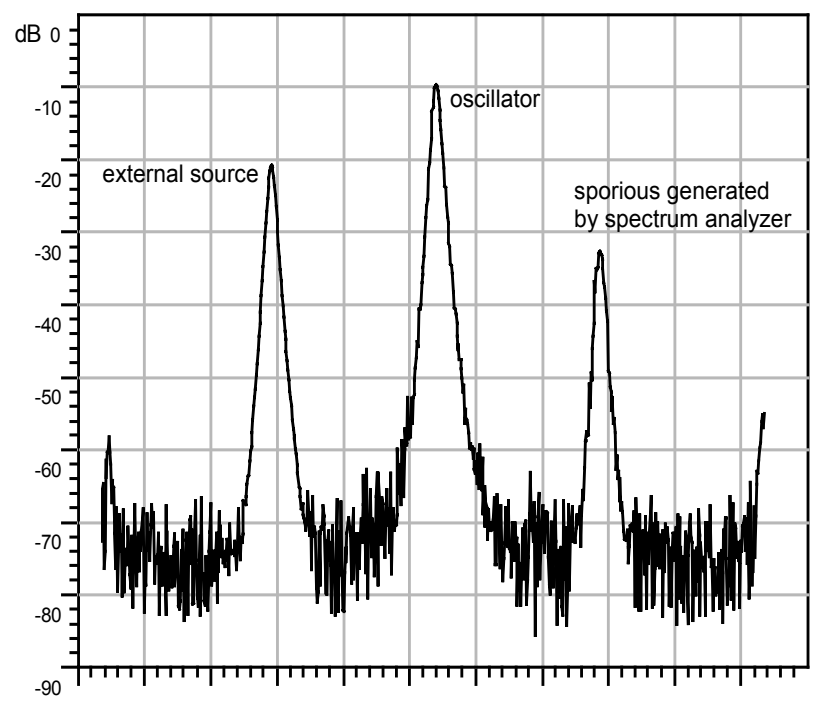

23.960 23.965 23.970 23.975 23.98023.985 23.990 23.995 24.000 24.005 24.010 GHz

Fig. 2. Injected signal is not within the locking range of the oscillator.

\section{Injection locking at the first harmonic}

In contrast to the described mechanism of injection locking above the injection locking in our case is different. The described mechanism requires that the injected signal is in the locking range of the free running oscillator's fundamental wave. Here, the oscillator is working as harmonic oscillator and the signal of interest is the first harmonic wave. The synchronisation signal, which is injected into the antenna, is also at the first harmonic of the oscillator, at a frequency of $24 \mathrm{GHz}$. So, what will happen by injecting the signal to this oscillator? The basic operation of a harmonic oscillator is that harmonics of the fundamental wave are generated by the nonlinearities of the active device. The only difference between a fundamental and a harmonic oscillator is the frequency where power is taken out of the oscillator. In our harmonic oscillator the first harmonic

$2 \omega_{0}=\omega_{h}$

is delivered at the output, where $\omega_{0}$ is the fundamental wave and $\omega_{h}$ is the first harmonic. Vice versa, the externally injected signal is handled.

The injected $24 \mathrm{GHz}$ signal $\omega_{i n j, h}$ is mixed down at the nonlinearities of the oscillator with its fundamental

$\omega_{i n j, h}-\omega_{0}=\omega_{i n j}$

where $\omega_{i n j}$ is the half of the injected signal. Now, we apply the theory described above to our oscillator.

In Fig. 2 the external source is not within the locking range of the free running oscillator or the amplitude of the external signal is not large enough. For these conditions no interaction between oscillator and external signal is observed.

In Fig. 3 the injected signal is at the edge of the oscillator's locking range. Significant interaction between the signals results in harmonics of the beat frequency. Decreasing 


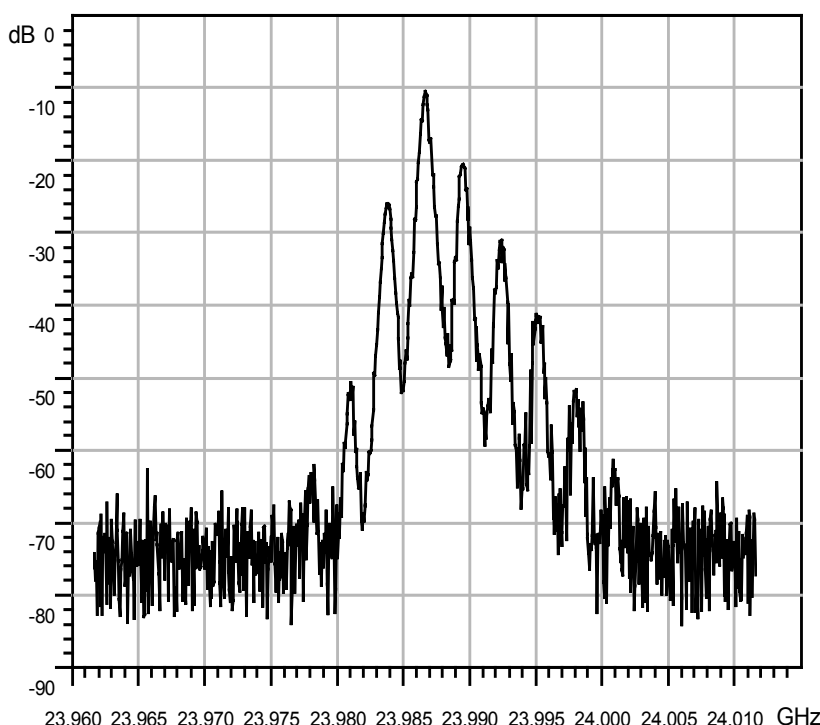

Fig. 3. Injected signal is very close to the locking range. Interference frequencies are produced.

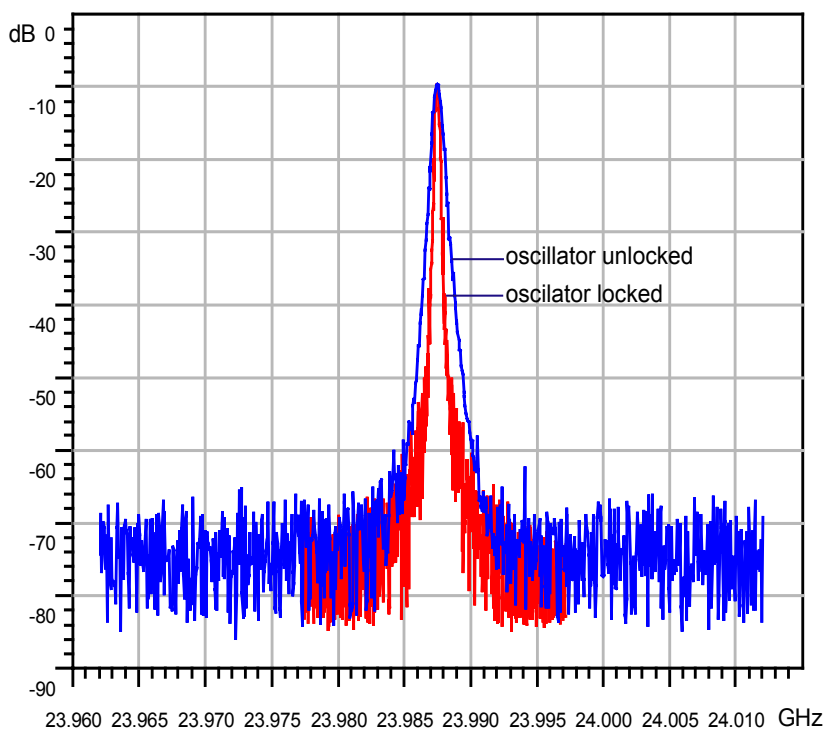

Fig. 4. Spectrum of free running and injection locked oscillator.

the frequency or increasing the power will lock the oscillator's frequency and phase to the injected signal (Fig. 4).

Another interesting point is the phase noise improvement due to the injection locking. The phase noise of the unlocked oscillator is $-84 \mathrm{dBc} @ 1 \mathrm{MHz}$, the phase noise of the locked oscillator is $-88 \mathrm{dBc} @ 1 \mathrm{MHz}$. The reduction of the phase noise can be analysed using the method given in Cao and York (1995). If we ignore AM noise, which is usually much less than the phase noise, the output voltage of an oscillator and the voltage of the injected signal can be written as

$V_{0}=A_{0} \cos \left(\omega_{0} t+\varphi_{0}(t)\right)$

$V_{i n j}=A_{i n j} \cos \left(\omega_{i n j} t+\varphi_{i n j}(t)\right)$

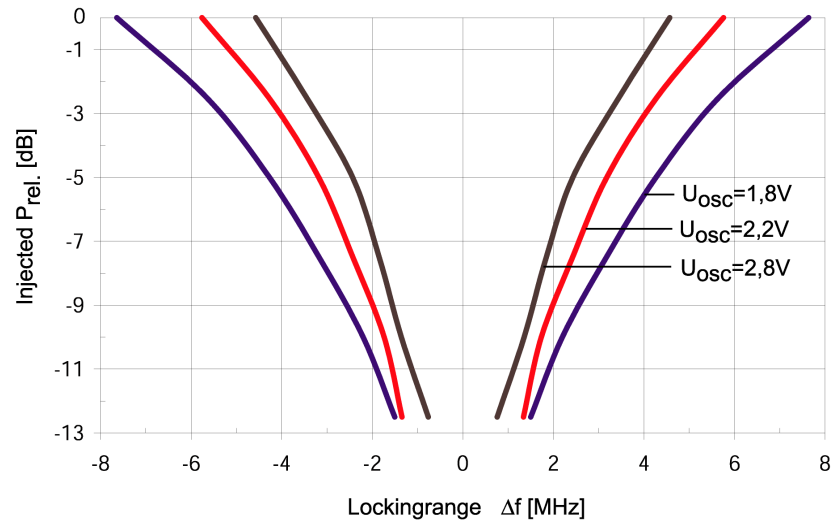

Fig. 5. Upper and lower edge of the locking range versus the power of the injected signal and DC bias of the oscillator's transistor.

With Adler's equation (3.1) and equation (4.3) and (4.4) it can be shown that the oscillator's phase variation follows the phase variations of the injected signal. As long as the oscillator is locked and the variations of the injected signal are within the locking range the phase variations follow

$\frac{d \varphi_{0}(t)}{d t}=\left(\omega_{i n j}-\omega_{0}\right)+\frac{d \varphi_{i n j}(t)}{d t}$,

i.e. $\varphi_{0}(t)$ and $\varphi_{i n j}(t)$ are synchronous within the locking bandwidth (where $\omega_{0} \omega_{i n j}=0$ ).

In Fig. 5 the dependencies of the injection locking parameters are plotted. The locking range $\delta f$ depends on the injected power of the external signal and the amplitude of the free running oscillator (indicated by the DC drain-sourcevoltage $\mathrm{U}_{\mathrm{osc}}$ applied to the transistor). The dependence of the locking range versus the injected power can easily be explained by analysing Adler's equation (3.1). Increasing the injected power results in a larger amplitude $\rho$. Increasing $\rho$ will increase $\Delta \omega_{m}$. Because $\Delta \omega_{m}$ is the half of the locking range $\omega_{0} \pm \Delta \omega_{m}$ the locking range becomes larger. On the other hand decreasing the oscillator voltage will decrease the output amplitude $\alpha$ and increase the locking range in the same way.

\section{Summary}

A harmonic free running oscillator at $24 \mathrm{GHz}$ was demonstrated. The phase/frequency stability of the oscillator was enhanced by injection locking at the first harmonic, i.e. at $24 \mathrm{GHz}$. The oscillator can be used for applications in the ISM-band. The mechanism of injection locking at the first harmonic could be explained by an analogy to the theory for fundamental injection locking. Furthermore, the dependencies of the locking range versus power levels of the oscillator and the injected signal could be predicted and were proven by measurements. 


\section{References}

Olbrich, M.: Development of a $24 \mathrm{GHz}$ Doppler radar module, Diplomarbeit, Technische Universität München, April 2002.

Adler, R.: A study of Locking Phenomena in Oscillators, Proc. of the IRE, vol. 34, pp. 351-358, June 1946.

Kurokawa, K.: Injection-Locking of a solid-state microwave oscillators, Proc. IEEE, vol. 61, pp.1386-1409, Oct. 1973.
York, R. A.: Nonlinear Analysis of Phase Relationships in quasiOptical Oscillator Arrays, Trans. IEEE MTT, vol. 41, no. 10, pp. 1799-1809, Oct. 1993.

Navarro, J. A. and Chang K.: Integrated Active Antennas and Spartial Power Combining, K. Chang, Wiley, ch. 12, 1996.

Cao, X. and York, R. A.: Phase Noise Reduction in Scanning Oscillator Arrays, MTT-S, WE3F-F2, 1995. 